\title{
Development of a robust degassing process for carbonated beverage using gas stripping
}

\author{
Carina Évora Gomes
}

Department of Process and Chemical Engineering, UCC

\section{Introduction}

The modern beverage industry works in a dynamic and very competitive market, with increasing sales but decreasing profit margins. Projected global sales for carbonated soft beverages are expected to reach $\$ 195$ billion by 2014 . Carbonated drinks are differentiated from other drinks by their "fizziness" (that comes from the dissolved carbon dioxide), which creates a "tingling" sensation in the mouth, and provides a unique taste sensation. They can be divided in non-alcoholic and alcoholic beverages. The non-alcoholic drinks are known as soft drinks and can be fruit based (orange, lemon and lime), cola type drinks (Pepsi, Coca cola), or others such as new energy drinks (like Red Bull), while alcoholic carbonated drinks include beer, cider, sparkling wines, etc. In order to maintain the flavour and the other quality attributes at the appropriate consumer level of appreciation, it is necessary to perform several quality control (QC) analyses. The most usual quality control tests performed are the sugar content (Brix), Acidity $(\mathrm{pH})$ and the total amount of acid in the drink (Titratable acidity). The presence of gas bubbles in the carbonated drink interferes with most of these tests and therefore the degassing (removal of carbon dioxide) of the beverage is essential to prevent the interference of gas bubbles with the quality control analysis, which could even damage the analytical equipment.

Current methods of degassing samples can be slow and fairly imprecise, which means that they may induce error and variability in the subsequent measurements, and can represent a burden on precious time availability.

An innovative Irish company (Somex Ltd) explored the opportunity to develop a robust and reliable degassing technique based on the principle of gas stripping; replacing a soluble gas $\left(\mathrm{CO}_{2}\right)$ with another gas (air) with a much lower solubility ( by bubbling air into the beverage ). A collaborative research project, partly funded by Enterprise Ireland, was undertaken at University College Cork to analyse the new degassing process in a scientific manner, to critically assess it against current alternatives, and to improve its robustness and efficiency. 


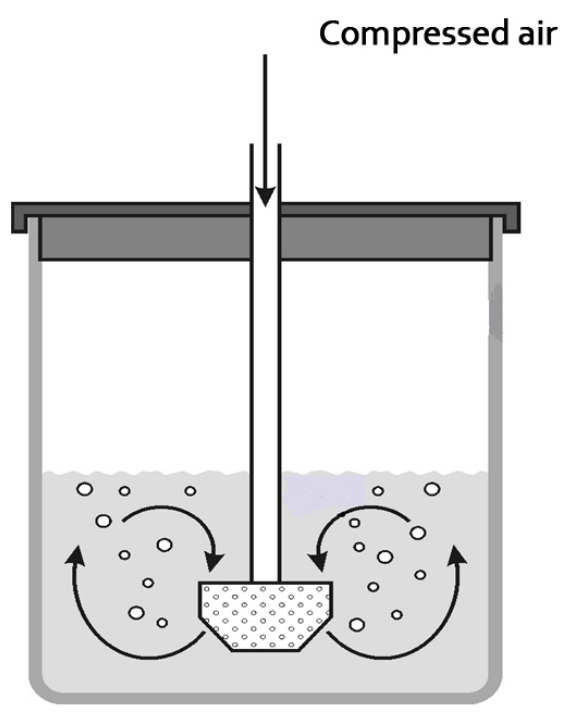

Figure 1: Schematic representation of the Somex gas stripping method of degassing with compressed air.

\section{Methods of research}

1. General analysis: Firstly, mathematical models were developed for degassing, the role of carbon dioxide presence in $\mathrm{pH}$ and titratable acidity measurements, and the stripping process of carbon dioxide by gas stripping with air, which were tested with "model" soft drinks (a water solution composed by the usual ingredients of cola-type beverages in their normal proportions) The interaction between the various acids and carbon dioxide was thus studied without the interference of sugars or other ingredients. This provided a fundamental understanding of the degassing process.

2. Degassing methods: Different degassing methods were compared, including the most usual standards in industry (vacuum, stirring, airstone, ultrasonication, filtration). Quality control parameters such as Brix, $\mathrm{pH}$ and titratable acidity were measured to assess the effect of the degassing technique on the values obtained in the QC readings and their variability. Tests were performed in model solutions and also in a variety of commercially available soft drinks and beers.

3. Stripping efficiency and nozzle design: The effectiveness of the removal of dissolved $\mathrm{CO}_{2}$ in a liquid by bubbling though an alternative gas (air) with a much lower solubility is dependant on the gas distributor or nozzles (gas sparger) design. This effect was also studied by using different alternative nozzle designs.

\section{Summary of the main results}

The model developed predicted that degassing would take 8 minutes, while experimental results showed that this was actually too conservative, and degassing with an appropri- 
ately developed efficient system can be undertaken in 4 minutes, or even less (depending on the nozzle design). This shows a substantial advantage over current methods which can take 10 to 30 minutes (in some individual cases, such as beers, even more than that).

The comparison tests performed with the different degassing methods also demonstrated that the new gas stripping process was much more robust; that is, the values measured afterwards of the QC parameters have a significantly lower variability than those that result from using other methods of degassing. It was therefore established that the new system is both more efficient (faster to use) and more effective (more precise and so less prone to introduce variability)

During the investigation, a variety of novel designs were developed for the gas sparging nozzle. The influence of the sparger design on the efficiency of the stripping process was quite evident, with different degassing times and inherent variability. It was also confirmed that this process degasses very fast and efficiently, with the optimum nozzles degassing in less than 4 minutes.

Using the optimum gas nozzle design, the validation of the results was was performed with a variety of commercial products, which proved that controlled gas stripping provided a more reliable and robust method of degassing and minimized variability in quality control for soft drinks. A second type of product considered was beer. For this more challenging case, (where alcohol may also evaporate during degassing) samples were degassed using the standard degassing of breweries (double filtration) and compared to the new gas stripping degassing. Subsequent quality control tests were performed measure $(\mathrm{pH}$, Titratable acidity, colour and alcohol content) for both degassing methods. The results showed that controlled gas stripping led to more accurate and repeatable results, even though it degassed in just 3 minutes.

The validation studies showed that very different types of carbonated beverages can be degassed very efficiently and effectively by the gas stripping technique.

\section{Conclusion}

This collaborative research between university and industry provided a strong case for the advantage of a new process developed by an Irish SME over existing alternatives and opened new avenues of development, both in terms of specific equipment design (nozzles) and of product applications (expanding from soft drinks to beer).

Carina Gomes is a PhD student at Department of Process and Chemical Engineering under the supervision of Dr. Jorge Oliveira. The author would like to acknowledge the contribution, support to this research from Brian O'Keeffe and funding from Somex Ltd and Enterprise Ireland. 